\title{
SIMBAD BIBLIOGRAPHY AND OTHER ASTRONOMICAL BIBLIOGRAPHIES
}

\author{
P. Dubois \\ Centre de Données de Strasbourg (CDS) \\ CNRS-URA $n^{\circ} 654$ \\ Observatoire de Strasbourg \\ 11 rue de l'Université \\ F-67000 STRASBOURG (France)
}

\begin{abstract}
SIMBAD is an astronomical database which includes bibliographical references. Some comparisons are made with other general or specialized astronomical bibliographies.
\end{abstract}

\section{INTRODUCTION}

SIMBAD is an astronomical database produced and updated by the Centre de Données de Strasbourg ( CDS ) at the Strasbourg Observatory ( France ). SIMBAD contains about 30 types of data, among them are bibliographical references.

Before comparing SIMBAD's bibliography with others, it must be noted that SIMBAD is organized by astronomical objects. In this way, the interrogation of SIMBAD is not made by keywords but by the names of the objects or a list of objects. Thus, SIMBAD contains only a few papers concerning theoretical work except if some objects are quoted in the paper. A thesaurus is replaced here by a list of objects. The following comparisons concern interrogations by object's name.

\section{COMPARISONS WITH GENERAL BIBLIOGRAPHIES}

\subsection{Fach-Informations Zentrum.}

A first comparison is made with the database produced by the Fach-Informations Zentrum at Karlsruhe ( FRG ). It concerns 8 objects and its bibliography for the years 1983 and 1984. 
Table I

$\begin{array}{ccc}\text { Name of object } & \begin{array}{c}\text { Number of references in } \\ \text { SIMBAD }\end{array} & \begin{array}{c}\text { Identical } \\ \text { FIZ }\end{array}\end{array}$

\begin{tabular}{lrrr} 
Alfa Lyr & 52 & 17 & 11 \\
Alfa Tau & 32 & 4 & 4 \\
T Tau & 42 & 9 & 7 \\
HZ Her & 45 & 24 & 18 \\
Zeta Cap & 16 & 6 & 5 \\
BL Lac & 49 & 29 & 27 \\
3C 273 & 116 & 46 & 29 \\
PSR 1937+214 & \multicolumn{54}{|c|}{$\quad$ Total } & $\mathbf{4 0 6}$ & 159 & $\underline{21}$ \\
\end{tabular}

The interrogation of the FIZ bibliography could give more references but some of them come from some confusion in the FIZ database. For instance, with the bibliography of alfa LYR ( known also as VEGA) FIZ gives also information concerning the spacecraft VEGA, or with the Zeta CAP bibliography FIZ provides information for other Barium stars.

2.2 Astronomy and Astrophysics Abstracts.

This comparison is made for the same objects and the year 1986.

Table II

Name of object

Number of references in
SIMBAD AAA

Identical

references

$\begin{array}{lrrr}\text { Alfa Lyr } & 25 & 12 & 7 \\ \text { Alfa Tau } & 22 & 11 & 7 \\ \text { T Tau } & 29 & 13 & 8 \\ \text { HZ Her } & 34 & 12 & 8 \\ \text { Zeta Cap } & 6 & 3 & 3 \\ \text { BL Lac } & 19 & 18 & 7 \\ \text { 3C 273 } & 69 & 46 & 16 \\ \text { PSR 1937+214 } & 21 & 11 & \frac{8}{64} \\ \quad \text { Total } & 225 & 126 & \end{array}$

For this comparison we may notice that there are more references in AAA for $T$ TAU and BL LAC because no differentiation is made between the star T TAU and the T TAU type stars, or between the object BL LAC and BL LAC type objects. We find also in AAA comparatively more references than in the FIZ bibliography and also more references which are not in SIMBAD. Another comparison made for the year 1985 for 18 other objects and concerning 329 references shows the same result. From these two comparisons we may expect that in 100 references, 80 could be retrieved by SIMBAD, 45 by AAA and 30 by FIZ. Only 25 references are in common. 
Therefore if objects are concerned, the bibliography retrieved by SIMBAD is clearly more important than what is found in other bibliographic databases even if they have reviewed a greater number of astronomical publications.

\section{COMPARISONS WITH SPECIALIZED BIBLIOGRAPHIES}

There exist many specialized bibliographies for different classes of astronomical objects. Here is a comparison with some of them.

3.1 Bibliographies using SIMBAD.

There exists a bibliography for planetary nebulae (Acker et al. 1984) and one for RR Lyr stars ( Heck 1988 ). SIMBAD.

In each of these bibliographies appear a few additional references not quoted in

\subsection{IUE bibliography ( NASA 1986 ).}

161 papers were checked; all are in SIMBAD. On the other hand, from SIMBAD it is not possible to know if these papers are based on IUE observations.

\subsection{Close binaries ( Herczeg 1986).}

In the first part of the bibliography which concerns objects, $90 \%$ of the papers (209 checked) are in SIMBAD. In the second part which concerns theoretical and general questions, $70 \%$ of the papers ( 40 were checked) are in SIMBAD.

\subsection{A Peculiar Newsletter.}

This bibliography cites information which is not always published and abstracts of preprints. For the latter, 100\% where found in SIMBAD (32 were checked for the year 1986 and 1987).

\subsection{Variable Stars.}

There exists a bibliography made by Huth and Wenzel (Wenzel 1981). A comparison is made with some of the stars of Tables I and II for the year 1980. The results are shown in Table III. 
$\underline{\text { Table III }}$

\begin{tabular}{lccc} 
Name of object & \multicolumn{2}{c}{ Number of references in } & Identical \\
& SIMBAD & HW & references \\
Alfa Lyr & 17 & 12 & 7 \\
Alfa Tau & 19 & 18 & 10 \\
T Tau & 11 & 17 & 3 \\
HZ Her & 32 & 29 & 15 \\
BL Lac & $\underline{4}$ & $\frac{1}{77}$ & $\underline{0}$ \\
Total & 83 & & 35
\end{tabular}

The two bibliographies have about the same number of references but only $25 \%$ are in common. This is explained by the fact that, on the one hand, the bibliography of variable stars uses many bulletins of Variable Stars Observers's Associations which are not taken into account in SIMBAD and on the other hand that SIMBAD has more references from the main journals.

\section{CONCLUSION}

This study based upon more than 1600 bibliographical references shows :

1) The difficulty in making an exhaustive survey of papers.

2) It may be preferable to locate theoretical subjects via general astronomical bibliographies using a thesaurus or an inverse file of the texts.

3) Specialized bibliographies of ten appear to be a duplication of the survey made by SIMBAD, at least for a major part. However all these bibliographies give some additional information not contained in SIMBAD.

4) If the search concerns objects, SIMBAD appears to be very efficient in any case and is of ten the most effective tool for retrieving a bibliography.

\section{REFERENCES}

-Acker A., Gleizes F., Chopinet M., Marcout J., Ochsenbein F., Roques J.M.: 1982 , Publ. Spec. CDS n³.

-Fernandez A., Lortet M.C. and Spite F.: 1983, Astron. and Astrophys. Supp. 52,1.

-Heck A.: 1988, Publ. Spec. CDS $n^{\circ} 11$.

-Herczeg T.J.: 1986, IAU Com. 42 Bibliography and Program Notes on Close Binaries $n^{\circ}$ 43. Ed. T.J. Herczeg .

-Lortet M.C. and Spite F.: 1986, Astron. and Astrophys. Supp. 64, 329.

-NASA : 1986, International Ultraviolet Explorer. Nasa Letter $n^{\circ} 30$.

-Wenzel W.: 1981, Bull. Inf. CDS n² 20, 105. 